\title{
Upconversion of a relativistic Coulomb field terahertz pulse to the near infrared
}

\author{
S. P. Jamison, ${ }^{1, \text { a) }}$ G. Berden, ${ }^{2}$ P. J. Phillips, ${ }^{3,4}$ W. A. Gillespie, ${ }^{4}$ and A. M. MacLeod ${ }^{5}$ \\ ${ }^{1}$ Accelerator Science and Technology Center, STFC Daresbury Laboratory, Warrington WA4 4AD, \\ United Kingdom \\ ${ }^{2}$ FOM Institute for Plasma Physics Rijnhuizen, Edisonbaan 14, 3439 MN Nieuwegein, The Netherlands \\ ${ }^{3}$ Central Laser Facility, STFC Rutherford Appleton Laboratory, Didcot OX11 OQX, \\ United Kingdom \\ ${ }_{5}^{4}$ Carnegie Laboratory of Physics, University of Dundee, Dundee DD1 4HN, United Kingdom \\ ${ }^{5}$ School of Computing and Engineering Systems, University of Abertay Dundee, Dundee DD1 $1 H G$, \\ United Kingdom
}

(Received 9 April 2010; accepted 14 May 2010; published online 10 June 2010)

\begin{abstract}
We demonstrate the spectral upconversion of a unipolar subpicosecond terahertz (THz) pulse, where the THz pulse is the Coulomb field of a single relativistic electron bunch. The upconversion to the optical allows remotely located detection of long wavelength and nonpropagating components of the $\mathrm{THz}$ spectrum, as required for ultrafast electron bunch diagnostics. The upconversion of quasimonochromatic $\mathrm{THz}$ radiation has also been demonstrated, allowing the observation of distinct sum- and difference-frequency mixing components in the spectrum. Polarization dependence of first and second order sidebands at $\omega_{\mathrm{opt}} \pm \omega_{\mathrm{THz}}$, and $\omega_{\mathrm{opt}} \pm 2 \omega_{\mathrm{THz}}$, respectively, confirms the $\chi^{(2)}$ frequency mixing mechanism. (C) 2010 American Institute of Physics. [doi:10.1063/1.3449132]
\end{abstract}

The Coulomb field of a subpicosecond relativistic electron bunch, in the vicinity of the electron bunch, has a temporal profile of a unipolar terahertz $(\mathrm{THz})$ pulse. Measurement of the spectrum of this Coulomb pulse can be used to characterize the temporal profile of the bunch. Measuring the spectrum radiated via mechanisms such as coherent transition radiation, ${ }^{1,2}$ coherent synchrotron radiation, ${ }^{3,4}$ or SmithPurcell radiation ${ }^{5}$ poses problems as the radiated spectrum spans the far infrared (IR) to mid-IR. These techniques thus seek to measure an extremely broadband spectrum, specifically including the long wavelength components, which are subject to strong diffraction limitations, and which are required for reliable temporal profile reconstruction, but which cannot propagate except through near-field diffraction effects.

Here we demonstrate, in a proof-of-principle experiment, the upconversion of the broadband Coulomb $\mathrm{THz}$ pulse to the near-IR through $\chi^{(2)}$ sum- and differencefrequency mixing. The upconversion allows the spectral information in long wavelength and zero-frequency field components to undergo effectively diffraction-free propagation to remote detectors. It also provides a narrowing of the relative bandwidth of the spectrum to be measured, enabling singleshot detection of the spectrum of $\mathrm{THz}$ pulses using optical spectrometers equipped with arrays of charge-coupled device (CCD) detectors.

While this demonstration is of particular interest for ultrafast relativistic particle beam diagnostics, the technique itself can clearly be applied more generally to the characterization of $\mathrm{THz}$ or mid-IR sources. We have carried out a second experiment that highlights this capability, with the upconversion and subsequent optical detection of quasimonochromatic $\mathrm{THz}$ pulses generated by a free-electron laser (FEL). The tunable FEL pulse allows the observation of

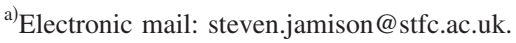

optical fields at the distinct sum- and difference-frequencies $\omega_{\mathrm{opt}} \pm \omega_{\mathrm{THz}}$. We have also observed the generation of second order sidebands at $\omega_{\mathrm{opt}} \pm 2 \omega_{\mathrm{THz}}$ which are attributed to a cascading of the $\chi^{(2)}$ frequency mixing process.

Experiments have been performed at the Free Electron Laser for Infrared eXperiments (FELIX) in Nieuwegein (The Netherlands). The experimental arrangement for the Coulomb field measurements is shown in Fig. 1. The optical probe, a quasimonochromatic Ti:S laser pulse [wavelength $\lambda=790 \mathrm{~nm}$, transform limited pulse duration $\tau=3$ ps full width at half maximum (FWHM)], was transported in free space over $30 \mathrm{~m}$ to the accelerator area. A half-wave plate and polarizer were used to orientate the polarization vector of the input beam before injection into the electron beamline. The electron bunch had an energy of $50 \mathrm{MeV}$, charge of 250 $\mathrm{pC}$, and an expected duration of 650 fs FWHM. ${ }^{6}$ A $0.5 \mathrm{~mm}$ $\langle 110\rangle$ orientated zinc telluride (ZnTe) crystal installed inside the electron beamline was the non-linear medium for frequency mixing. The crystal is transversely offset from the electron beam trajectory by $2 \mathrm{~mm}$, intercepting the Coulomb field but not the bunch itself. When the optical probe and Coulomb pulse pass through the ZnTe in synchronism they undergo frequency mixing and upconversion of the $\mathrm{THz}$ spectrum. Immediately after the $\mathrm{ZnTe}$ crystal, the probe is
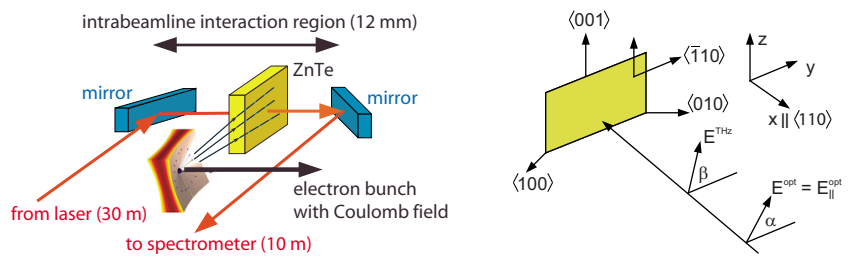

FIG. 1. (Color online) The experimental arrangement for Coulomb field spectral upconversion and the definition of the crystal and reference frames. The probe input and Coulomb $\mathrm{THz}$ pulse propagate along the $\mathrm{ZnTe}\langle\overline{1} \overline{1} 0\rangle$ direction, with polarization defined by the angles $\alpha$ and $\beta$ with respect to the $\langle\overline{1} 10\rangle$ direction. 


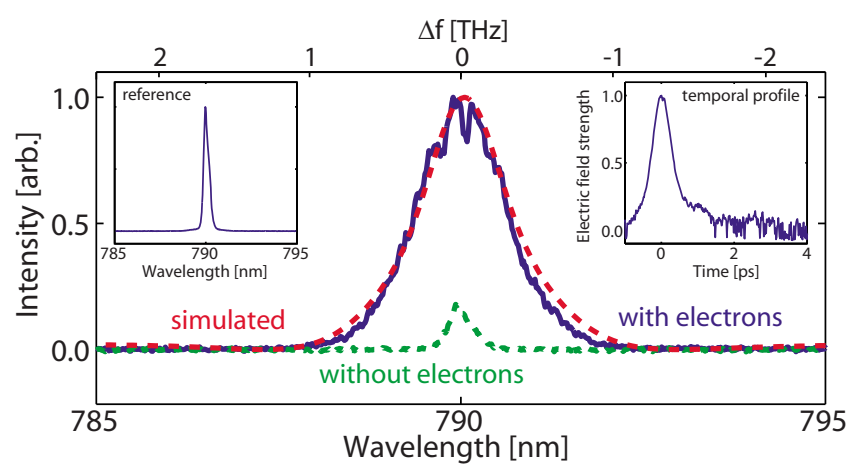

FIG. 2. (Color online) The measured and predicted upconverted Coulomb $\mathrm{THz}$ spectrum, together the spectrum observed without the Coulomb pulse. The probe laser reference spectrum and the Coulomb temporal profile are shown in the insets.

reflected from the FEL beamline and transported $10 \mathrm{~m}$ from the accelerator area for optical spectral characterization (grating spectrometer with gated CCD camera). A sequence of waveplates and polarizers is used to suppress residual birefringence and restrict measurement of the optical spectrum to that with polarization orthogonal to the input probe.

Figure 2 shows the spectrum observed when the probe pulse and the Coulomb $\mathrm{THz}$ pulse were temporally coincident, along with the spectrum observed in the absence of electrons. A reference spectrum for the laser is also shown, and was produced by rotating the analyzing polarizer by $3^{\circ}$ from the optimum extinction. That the changes in the spectrum induced by the Coulomb $\mathrm{THz}$ pulse are not the result of a rotation in the polarization of the probe beam, as in the electro-optic or Pockels effect, is apparent from the broader extent of the upconverted spectrum when compared to the probe reference.

We attribute the generation of a perpendicularly polarized optic field, with increased spectral width compared to the original probe, to $\chi^{(2)}$ frequency mixing between the probe and Coulomb $\mathrm{THz}$ pulse. The nonlinear polarization source term for $\chi^{(2)}$ mixing, for specific optical and $\mathrm{THz}$ frequencies, is $\tilde{p}_{k}^{\mathrm{opt}}(\omega, \Omega)=\epsilon_{0} \sum_{i j} \chi_{i j k}^{(2)} \widetilde{E}_{i}^{\mathrm{THz}}(\Omega) \widetilde{E}_{j}^{\mathrm{opt}}(\omega-\Omega)$ where $i, j, k$ refer to the crystal axes. ${ }^{7,8}$ The full source term at optical frequency $\omega$ is obtained from the integral over all THz frequencies, $\widetilde{P}_{k}^{\text {opt }}(\omega)=\int \widetilde{p}_{k}^{\text {opt }}(\omega, \Omega) \mathrm{d} \Omega$. For crystals with ZnTe symmetry $\chi_{i j k}^{(2)}=\chi^{(2)}$ for $i \neq j \neq k$ and zero otherwise. In the experiments here, the crystal is orientated with the $\mathrm{THz}$ and probe beam propagation direction parallel to the $\langle\overline{1} \overline{1} 0\rangle$ direction as shown in Fig. 1, for which $\widetilde{p}_{x}=0$ identically. Hence, we are free to consider only the $y$-axis and z-axis components of the polarization,

$$
\begin{aligned}
{\left[\begin{array}{c}
\tilde{p}_{y}(\omega, \Omega) \\
\widetilde{p}_{z}(\omega, \Omega)
\end{array}\right]=} & -\epsilon_{0} \chi^{(2)}\left[\begin{array}{cc}
\widetilde{E}_{z}^{\mathrm{THz}}(\Omega) & \widetilde{E}_{y}^{\mathrm{THz}}(\Omega) \\
\widetilde{E}_{y}^{\mathrm{THz}}(\Omega) & 0
\end{array}\right] \\
& \times\left[\begin{array}{c}
\widetilde{E}_{y}^{\mathrm{opt}}(\omega-\Omega) \\
\widetilde{E}_{z}^{\mathrm{opt}}(\omega-\Omega)
\end{array}\right] .
\end{aligned}
$$

In general, the source polarization of Eq. (1) will have components both parallel and perpendicular to the optical probe, for which we adopt the notation of

$$
\widetilde{p}_{\|}(\omega, \Omega)=-F_{\|} \widetilde{E}^{\mathrm{THz}}(\Omega) \widetilde{E}_{\|}^{\mathrm{opt}}(\omega-\Omega),
$$

$$
\tilde{p}_{\perp}(\omega, \Omega)=-F_{\perp} \widetilde{E}^{\mathrm{THz}}(\Omega) \widetilde{E}_{\|}^{\mathrm{opt}}(\omega-\Omega),
$$

where the geometrical factors $F_{\|}, F_{\perp}$ can be found from Eq. (1) through a coordinate transformation aligning the frame with the optical probe polarization. $\widetilde{E}^{\mathrm{THz}}(\Omega)$ without coordinate subscript refers to the magnitude of the $\mathrm{THz}$ field as shown in Fig. 1. In this probe frame, with integration over all $\mathrm{THz}$ frequencies, the solution to the wave-equation for the optical spectrum (in the small signal limit), is simply ${ }^{7,8}$

$$
\widetilde{E}_{\|}^{\text {out }}(\omega)=\widetilde{E}_{\|}^{\text {opt }}(\omega)-i g F_{\|} \int_{-\infty}^{\infty} \mathrm{d} \Omega \zeta(\Omega) \widetilde{E}^{\mathrm{THz}}(\Omega) \widetilde{E}_{\|}^{\mathrm{opt}}(\omega-\Omega),
$$

$\widetilde{E}_{\perp}^{\text {out }}(\omega)=-i g F_{\perp} \int_{-\infty}^{\infty} \mathrm{d} \Omega \zeta(\Omega) \widetilde{E}^{\mathrm{THz}}(\Omega) \widetilde{E}_{\|}^{\text {opt }}(\omega-\Omega)$,

where $g$ denotes propagation constants that do not affect the discussion here. The phase matching is described by $\zeta(\Omega)$, which for our experimental parameters can be approximated by the crystal thickness. The generation of both sum- and difference-frequency optical fields arises from the positive and negative $\mathrm{THz}$ frequencies, with $\widetilde{E}^{\mathrm{THz}}(-|\Omega|)$ $=\left[\widetilde{E}^{\mathrm{THz}}(|\Omega|)\right]^{*}$.

For the Coulomb field upconversion experiment, the polarization geometry is fixed and the expected spectrum is given by Eq. (5). For a comparison between the measured spectrum and that expected from the upconversion mechanism, the Coulomb field spectrum is required. Here we are able to be guided by past experiments measuring the FELIX electron bunch temporal profile using the related technique of "electro-optic temporal decoding." 6 The spectrum of the $\mathrm{THz}$ Coulomb field expected in the present experiments is inferred, through Fourier transform, from the directly measured Coulomb field temporal profile of the earlier experiments (see inset of Fig. 2). The spectrum of the probe laser electric field was obtained from the measured reference intensity spectrum, $E^{\mathrm{opt}}(\omega)=\left[I^{\mathrm{opt}}(\omega)\right]^{1 / 2}$. The convolution integral of Eq. (5) was evaluated with these (complex) optical probe and $\mathrm{THz}$ electric field spectra, and the resulting simulation is shown in Fig. 2. A remarkably good agreement between calculation and experiment is obtained. While the theory-experiment comparison has been made possible by independent knowledge of the Coulomb temporal profile, for future applications we propose the converse, with the measured spectrum used to infer temporal information, as is common in electron bunch diagnostics. ${ }^{1-5}$

In a separate experiment, we have verified that the optical fields are produced at $\omega_{\mathrm{opt}} \pm \omega_{\mathrm{THz}}$, according to Eqs. (4) and (5), by observing the upconversion of a quasimonochromatic THz FEL source which, together with a monochromatic optical probe, allows the sum and difference frequency mixing contributions to be observed distinctly. A similar observation of the first order sidebands has previously been reported by Zaks et $a l .{ }^{9}$ Here, we were also able to observe a higher-order cascading process, ${ }^{10}$ whereby the first order sidebands themselves undergo mixing with the $\mathrm{THz}$ field, producing second order sidebands at $\omega_{\mathrm{opt}} \pm 2 \omega_{\mathrm{THz}}$. We have measured the intensities of the first and second order sidebands as a function of FEL THz polarization, and compared them with that expected for the $\chi^{(2)}$ mixing process. 

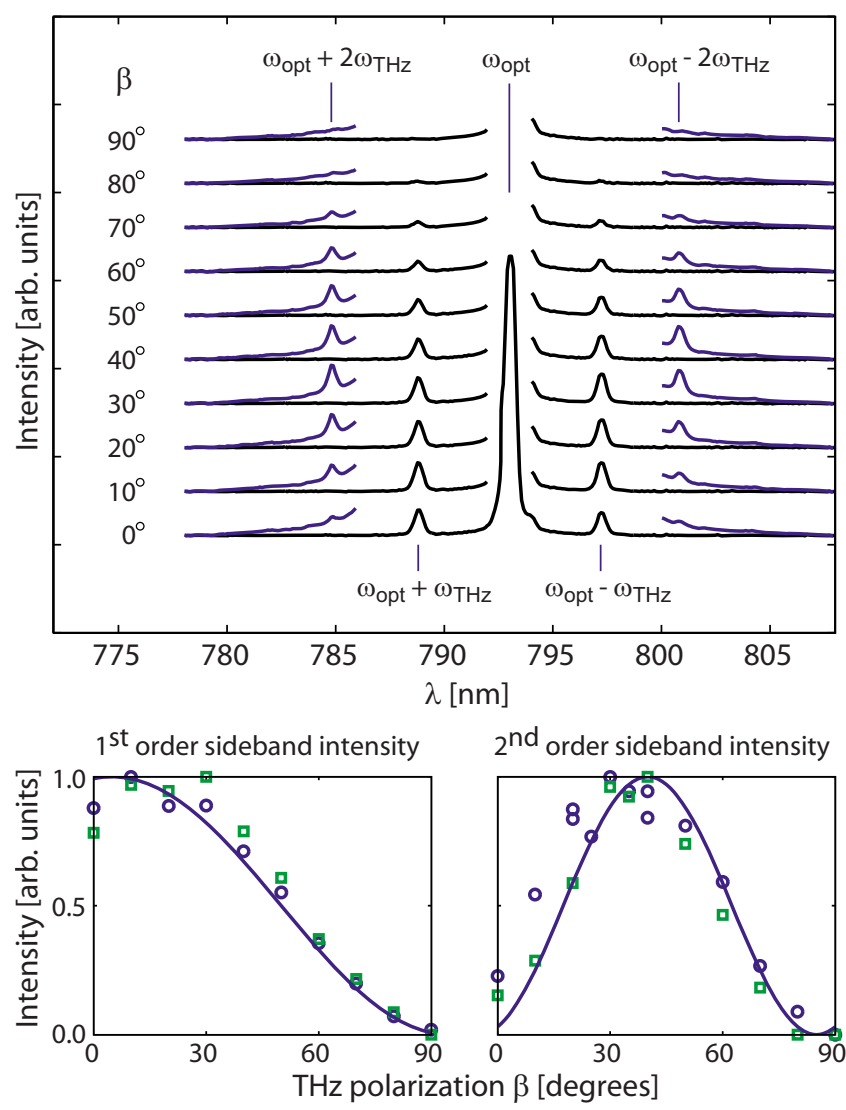

FIG. 3. (Color online) Top: measured spectra showing sidebands as a function of $\mathrm{THz}$ polarization $\beta$. Bottom: sum-frequency $(\bigcirc)$ and differencefrequency $(\square)$ sideband intensity as function of $\mathrm{THz}$ polarization, together with that predicted by $\chi^{(2)}$ frequency mixing (solid line).

For this experiment, the FEL pulse was focused with a $\mathrm{f}_{\text {eff }}=150 \mathrm{~mm}$ parabolic mirror on to a $0.5 \mathrm{~mm}$ thick $\langle 110\rangle$ ZnTe crystal orientated as in Fig. 1. The polarization of the $\lambda=793 \mathrm{~nm}$ optical probe was orientated with $\alpha=0\left(\tilde{E}_{y}^{\mathrm{opt}}\right.$ $\left.=\widetilde{E}_{\|}^{\text {opt }}, \widetilde{E}_{z}^{\text {opt }}=0\right)$. We sought to observe $\widetilde{E}_{z}^{\text {opt }}$, with the analyzing polarizer set to extinguish the optical probe; this extinction was limited by uncompensated birefringence. The optical probe and $\mathrm{THz}$ pulse were combined for collinear propagation through the ZnTe using an indium tin oxide dichroic beam splitter. In the experiments presented in Fig. 3 the FEL was tuned to $\lambda=150 \mu \mathrm{m}(2.0 \mathrm{THz})$, with a bandwidth of $2.4 \mu \mathrm{m}$ and a pulse energy of approximately $2 \mu \mathrm{J}$.

For varying $\mathrm{THz}$ polarization, the intensity of the sidebands will be determined by the geometrical factors $F_{\|}, F_{\perp}$. Referring to Eq. (1) and Fig. 1 with $\widetilde{E}_{y}^{\mathrm{THz}} \propto \cos \beta$ and $\widetilde{E}_{z}^{\mathrm{THz}}$ $\propto \sin \beta$, the parallel and perpendicular polarized contributions to the first order side band source term are given by $F_{\|}^{(1)}=\sin \beta$ and $F_{\perp}^{(1)}=\cos \beta$. The first order sidebands will then have expected intensity dependence $I_{\perp}^{(1)}=\cos ^{2} \beta$. Calculation of the intensity and polarization dependence of the second order sidebands requires an extension of the wave equation solution of Eqs. (4) and (5) beyond the weak-signal limit. ${ }^{10}$ Provided the $\mathrm{THz}$ intensity is not so great as to lead to significant third-order sidebands, the polarization of second order sidebands can be described through reapplication of the source term of Eq. (1), but taking the optical input polarization as that of the first order sidebands. This leads to the expectation of second order sideband intensity that varies as $I_{\perp}^{(2)}=\sin ^{2} 2 \beta$.

Figure 3 shows examples of the optical spectra observed for varying FEL polarization, with the trends in the first and second order sideband intensities clearly visible. The lower plots of Fig. 3 show the intensity variation in the sidebands together with the polarization dependence expected from the $\chi^{(2)}$ mechanism. The experimental data suggests a small $5^{\circ}$ misalignment in the crystal orientation or FEL polarization, and this angular offset has been included in the displayed calculation. From the very good agreement between the observed and predicted polarization dependence, we conclude that $\chi^{(2)}$ frequency mixing is responsible for both first and second order sidebands.

In conclusion, we have demonstrated upconversion of broadband unipolar $\mathrm{THz}$ pulses to the visible, enabling transport of highly diffracting and nonpropagating spectral components to remote detectors, and the detection of $\mathrm{THz}$ spectra with optical CCDs. The $\chi^{(2)}$ mixing description of the upconversion has been confirmed by observations of quasimonochromatic $\mathrm{THz}$ upconversion, and its polarization dependence. We believe the $\mathrm{THz}$ upconversion described here will find application in ultrafast electron bunch diagnostics, and more generally in single-shot $\mathrm{THz}$ spectroscopy.

Upconverted $\mathrm{THz}$ spectra have been measured at the FOM Institute for Plasma Physics Rijnhuizen, which is financially supported by NWO. The authors thank the FELIX staff for excellent support.

${ }^{1}$ S. Casalbuoni, B. Schmidt, P. Schmüser, V. Arsov, and S. Wesch, Phys. Rev. ST Accel. Beams 12, 030705 (2009).

${ }^{2}$ P. Kung, H.-C. Lihn, H. Weidemann, and D. Bocek, Phys. Rev. Lett. 73, 967 (1994).

${ }^{3}$ H.-W. Hübers, A. Semenov, K. Holldack, U. Schade, G. Wüstefeld, and G. Gol'tsman, Appl. Phys. Lett. 87, 184103 (2005).

${ }^{4}$ G. Andonian, A. Cook, M. Dunning, E. Hemsing, G. Marcus, A. Murokh, S. Reiche, D. Schiller, J. B. Rosenzweig, M. Babzien, K. Kusche, and V. Yakimenko, Phys. Rev. ST Accel. Beams 12, 030701 (2009).

${ }^{5}$ V. Blackmore, G. Doucas, C. Perry, B. Ottewell, M. F. Kimmitt, M. Woods, S. Molloy, and R. Arnold, Phys. Rev. ST Accel. Beams 12, 032803 (2009).

${ }^{6}$ G. Berden, S. P. Jamison, A. M. MacLeod, W. A. Gillespie, B. Redlich, and A. F. G. van der Meer, Phys. Rev. Lett. 93, 114802 (2004).

${ }^{7}$ Y. R. Shen, The Principles of Nonlinear Optics (Wiley, New York, 1984).

${ }^{8}$ S. P. Jamison, A. M. MacLeod, G. Berden, D. A. Jaroszynski, and W. A. Gillespie, Opt. Lett. 31, 1753 (2006).

${ }^{9}$ B. Zaks, J. Heyman, D. Stehr, D. Allen, N. Coates, and M. Sherwin, IEEE Proceedings of the 33rd International Conference on Infrared, Millimeter, and Terahertz Waves (IEEE, New York, 2008).

${ }^{10}$ S. P. Jamison, Appl. Phys. B: Lasers Opt. 91, 241 (2008). 